\title{
Silicon-Based Optical Mirror Coatings for Ultrahigh Precision Metrology and Sensing
}

\author{
J. Steinlechner, ${ }^{1,2}$ I. W. Martin, ${ }^{1, *}$ A. S. Bell, ${ }^{1}$ J. Hough, ${ }^{1}$ M. Fletcher, ${ }^{1}$ P. G. Murray, ${ }^{1}$ R. Robie, ${ }^{1}$ \\ S. Rowan, ${ }^{1}$ and R. Schnabel ${ }^{2}$ \\ ${ }^{1}$ SUPA, School of Physics and Astronomy, University of Glasgow, Glasgow G12 8QQ, Scotland \\ ${ }^{2}$ Institut für Laserphysik and Zentrum für Optische Quantentechnologien, \\ Universität Hamburg, Luruper Chaussee 149, Hamburg 22761, Germany
}

(Received 31 January 2018; revised manuscript received 10 March 2018; published 29 June 2018)

\begin{abstract}
Thermal noise of highly reflective mirror coatings is a major limit to the sensitivity of many precision laser experiments with strict requirements such as low optical absorption. Here, we investigate amorphous silicon and silicon nitride as an alternative to the currently used combination of coating materials, silica, and tantala. We demonstrate an improvement by a factor of $\approx 55$ with respect to the lowest so far reported optical absorption of amorphous silicon at near-infrared wavelengths. This reduction was achieved via a combination of heat treatment, final operation at low temperature, and a wavelength of $2 \mu \mathrm{m}$ instead of the more commonly used $1550 \mathrm{~nm}$. Our silicon-based coating offers a factor of 12 thermal noise reduction compared to the performance possible with silica and tantala at $20 \mathrm{~K}$. In gravitational-wave detectors, a noise reduction by a factor of 12 corresponds to an increase in the average detection rate by three orders of magnitude $\left(\approx 12^{3}\right)$.
\end{abstract}

DOI: $10.1103 /$ PhysRevLett.120.263602

Introduction.-Since 2015, several gravitational-wave (GW) signals from binary black hole mergers and a binary neutron-star merger were detected by the Advanced LIGO and Advanced Virgo GW detectors [1-5]. Such detectors are kilometer-scale laser interferometers which measure the separation of suspended, highly reflective (HR) coated mirrors. Coating thermal noise [6,7], which is a motion of the mirrors' surfaces due to the mirrors' temperatures, forms a major barrier to the detectors' design sensitivity. Future detectors such as the Einstein Telescope [8,9] and upgrades to Advanced LIGO [10] are planned to be operated at cryogenic temperatures to reduce thermal noise. Optical resonators for laser stabilization, e.g., for optical atomic clocks [11-13], are another important application for HR mirror coatings with low thermal noise at low temperatures. However, due to a temperature dependence of material properties [14], cooling alone does not solve the thermal-noise problem.

HR coatings consist of alternating layers of materials with a high and a low refractive index $n$. In the simplest case, the layers are a quarter of the coating's design wavelength in optical thickness, which is $n$ multiplied by the geometrical thickness $t$. Silica $\left(\mathrm{SiO}_{2}\right)$ and tantala $\left(\mathrm{Ta}_{2} \mathrm{O}_{5} x\right)$, which are widely used coating materials $[13,15]$, meet the requirements on the optical absorption at the ppm

Published by the American Physical Society under the terms of the Creative Commons Attribution 4.0 International license. Further distribution of this work must maintain attribution to the author(s) and the published article's title, journal citation, and DOI. $\left(10^{-6}\right)$ level [16]. However, the mechanical loss of these materials limits the thermal noise performance of mirrors at room temperature and shows evidence of a significant increase on cooling $[17,18]$. In advanced GW detectors, $\mathrm{Ta}_{2} \mathrm{O}_{5}$ is doped with titania $\left(\mathrm{Ti}: \mathrm{Ta}_{2} \mathrm{O}_{5}\right)$ as there is evidence of the doping slightly decreasing mechanical loss $[16,18,19]$. Coating $A$ in Fig. 1 shows the properties of a coating made of the materials used in Advanced LIGO at room temperature and designed for $1064 \mathrm{~nm}$.

Crystalline silicon $(c-\mathrm{Si})$ is an attractive mirror-substrate material due to a very low mechanical loss at low temperatures [20,21], zero thermal expansion at 120 and $20 \mathrm{~K}$ $[22,23]$, and high thermal conductivity. Previously, the telecommunication wavelength of $1.55 \mu \mathrm{m}$ was proposed for future, cryogenically operated $\mathrm{GW}$ detectors using $c-\mathrm{Si}$ mirrors $[9,23,24]$. The combination of $c$-Si and $1.55 \mu \mathrm{m}$ is already used in cavities for laser stabilization. Coating $B$ in Fig. 1 shows the performance of a coating made of Advanced LIGO materials, designed for $1.55 \mu \mathrm{m}$, cooled to $20 \mathrm{~K}$, and on a $c$-Si substrate instead of silica. The thermal noise improvement is only 50\%. Throughout this Letter, we use coating $B$ as a thermal-noise reference against which to compare the coatings we propose.

Crystalline AlGaAs coatings show very low thermal noise [25] and low optical absorption [26,27]. Such coatings can be used for coating thermal-noise reduction in laser stabiliztion systems. However, currently they are not available in the large diameter required for application in GW detectors [26]. A general advantage of amorphous coatings over crystalline AlGaAs coatings is that they can be sputtered directly onto suitable mirror substrates, while 


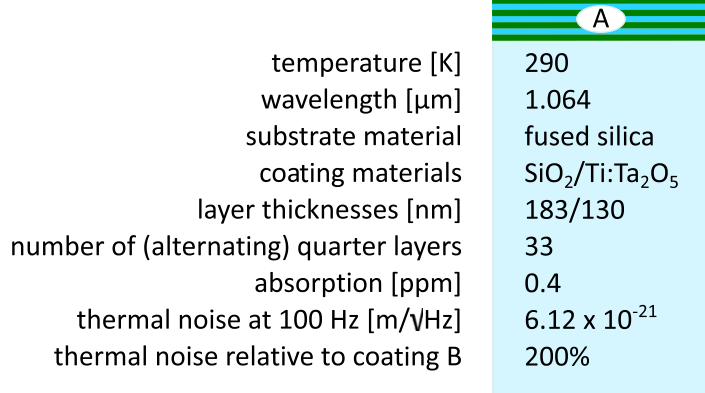

\begin{tabular}{l}
\hline \multicolumn{1}{c|}{$\mathrm{B}$} \\
\hline 20 \\
1.55 \\
silicon \\
$\mathrm{SiO}_{2} / \mathrm{Ti}^{\mathrm{T}} \mathrm{Ta}_{2} \mathrm{O}_{5}$ \\
$258 / 189$ \\
34 \\
1.7 \\
$3.11 \times 10^{-21}$ \\
$100 \%$ \\
(reference)
\end{tabular}

\begin{tabular}{l}
\hline $\mathrm{D}$ \\
\hline 20 \\
2.0 \\
silicon \\
$\mathrm{aSi} / \mathrm{SiN}$ \\
$143 / 179$ \\
22 \\
27 \\
$2.56 \times 10^{-22}$ \\
$8 \%$
\end{tabular}

FIG. 1. Schematics of the coatings discussed in this Letter. Coating $A$ is based on materials and parameters used in Advanced LIGO (at room temperature) but uses a simplified design with quarter layers only. The (integer) number of quarter layers is chosen for all coatings to have a similar reflectivity $(\approx 99.997 \%)$. Coating $B$ is the best coating that can be realized at $20 \mathrm{~K}$ with the coating materials used in Advanced LIGO. Coating $C$ shows the expected thermal noise reduction at $20 \mathrm{~K}$, when $\mathrm{Ti}_{\mathrm{T}} \mathrm{Ta}_{2} \mathrm{O}_{5}$ is replaced by low-loss $a$-Si presented here. Coating $D$ represents the coating proposed in this work. For all calculations, a beam radius of $6 \mathrm{~cm}$ was used. Table I shows the mechanical losses used for all thermal noise calculations.

AlGaAs coatings require a transfer from the lattice matched substrates, on which they are grown, to suitable substrates for the experiments.

A promising alternative to $\mathrm{Ta}_{2} \mathrm{O}_{5}$ is amorphous silicon (a-Si), which has much lower mechanical loss $[28,29]$ and a higher $n$ (see Table I). A higher index contrast between the coating materials allows fewer layers to provide the same reflectivity. This results in a direct thermal-noise reduction due to a thinner coating. However, $a$-Si has a significantly higher optical absorption than $\mathrm{Ta}_{2} \mathrm{O}_{5}$ at $1064 \mathrm{~nm}$ [30]. The absorption reduces at $1.55 \mu \mathrm{m}$ [31] but is still far above the requirements for GW detectors.

In this Letter, we present a reduction in optical absorption of $a$-Si by a factor of 55 compared to the best previous result [36]. This absorption improvement, which results from postdeposition heat treatment, a cryogenic operation temperature, and moving to a wavelength of $2 \mu \mathrm{m}$, makes the use of $a$-Si in HR coatings for precision measurements realistic.

The use of $a$-Si as a high-index material opens new possibilities for low-index partner materials with $n<n_{\mathrm{Si}}$, e.g., silicon nitride $(\mathrm{SiN})$ [37,38]. SiN has very low mechanical loss at low temperatures [32,33] and a low optical absorption compared to $a$-Si at $1.55 \mu \mathrm{m}$ [39], which we find to reduce further when moving to $2 \mu \mathrm{m}$. We show that an $a-\mathrm{Si} / \mathrm{SiN}$ coating at $20 \mathrm{~K}$ would have an optical

TABLE I. Mechanical loss $\phi$ and refractive index $n$ used for all wavelengths considered.

\begin{tabular}{lcccc}
\hline \hline & \multicolumn{4}{c}{ Mechanical loss $\phi \times 10^{-4}$} \\
\cline { 2 - 5 } Temperature [K] & $\mathrm{SiO}_{2}$ & $\mathrm{Ti}^{2} \mathrm{Ta}_{2} \mathrm{O}_{5}$ & $a-\mathrm{Si}^{\mathrm{a}}$ & $\mathrm{SiN}$ \\
\hline 290 & $0.4[16]$ & $2.4[16]$ & 0.2 & $0.8[32,33]$ \\
120 & $1.7[17]$ & $3.3[34]$ & $\leq 0.5{ }^{\mathrm{b}}$ & $0.2[32,33]$ \\
20 & $7.8[17]$ & $8.6[34]$ & $\leq 0.2^{\mathrm{b}}$ & $0.1[32,33]$ \\
$n$ & 1.5 & 2.05 & 3.65 & $2.17[35]$ \\
\hline \hline
\end{tabular}

${ }^{a}$ Our measurements.

${ }^{\mathrm{b}}$ Upper limit from sample without heat treatment. absorption of $27 \mathrm{ppm}$ at $2 \mu \mathrm{m}$. Thermal-noise amplitude spectral density of this coating would be a factor of 12 lower than that of an equivalently reflective $\mathrm{SiO}_{2} / \mathrm{Ta}_{2} \mathrm{O}_{5}$ coating at $1.55 \mu \mathrm{m}$ and $20 \mathrm{~K}$.

Optical absorption of a-Si.-We measured the absorption of an $a-\mathrm{Si} / \mathrm{SiO}_{2} \mathrm{HR}$ multilayer coating and an $a-\mathrm{Si}$ single-layer coating. The absorption of the HR coating was measured at the design wavelength of $1.55 \mu \mathrm{m}$. The singlelayer coating, with a design thickness of $1 \mu \mathrm{m}$, was measured at wavelengths of 1.55 and $2 \mu \mathrm{m}$. This enabled us to directly compare the absorption of $a-\mathrm{Si}$ at two wavelengths. Measuring the HR coating and the single layer both at $1.55 \mu \mathrm{m}$ enabled us to compare the performance of $a-\mathrm{Si}$ in both coating types (assuming that the optical absorption of the $\mathrm{SiO}_{2}$ layers is negligible, as previously observed [40]). The coatings were deposited on Corning 7980 fused silica substrates [41,42] by Tafelmaier Dünnschicht Technik GmbH [43] using reactive low voltage ion plating (RLVIP) [44] and a deposition temperature of about $250{ }^{\circ} \mathrm{C}$.

The optical absorption of the coatings was measured under normal incidence using photothermal commonpath interferometry [45]. The absorption was measured as a function of heat treatment temperature as shown in Fig. 2(a), where the samples were heat treated for $1 \mathrm{~h}$ in air postdeposition [46]. The optimum temperature was found to be $500{ }^{\circ} \mathrm{C}$.

The values in Fig. 2(a) are normalized to incident power and neither take into account surface reflection nor thin film interference. However, both must be considered to interpret the results and to predict the performance of an $a-\mathrm{Si} / \mathrm{SiO}_{2}$ HR coating. To do this, the thickness and refractive index of the single-layer coating were required. We measured its transmission at 1.55 and $2 \mu \mathrm{m}$ and matched it to a simulated reflection spectrum using [47]. For heat treatment up to $600^{\circ} \mathrm{C}$, the best agreement between measurement and simulation occurred for $t=1003 \mathrm{~nm}$ and $n=3.65$. These parameters were used to calculate the 

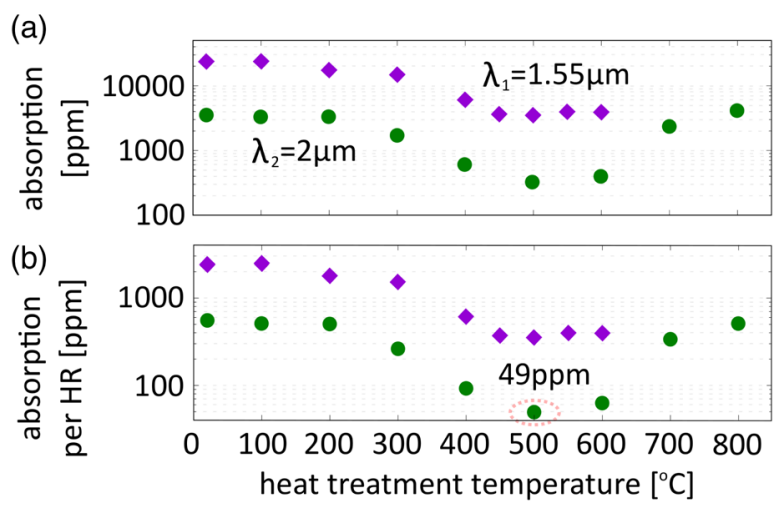

FIG. 2. (a) Optical absorption of our $1 \mu \mathrm{m}$ thick $a$-Si layer at $1.55 \mu \mathrm{m}$ (purple diamond) and at $2 \mu \mathrm{m}$ (green circle) as a function of postdeposition heat treatment temperature. The measurements were corrected for an absorption contribution from the substrate. (b) Optical absorption rescaled to the absorption expected in an $a-\mathrm{Si} / \mathrm{SiO}_{2} \mathrm{HR}$ coating. The minimum absorption (after heat treatment at $500{ }^{\circ} \mathrm{C}$ ) corresponds to an extinction coefficient of $k=6.25 \times 10^{-4}$ at $1.55 \mu \mathrm{m}$ and $8.6 \times 10^{-5}$ at $2 \mu \mathrm{m}$.

laser-power distribution in the single-layer coating, enabling the optical absorption of an $a-\mathrm{Si} / \mathrm{SiO}_{2} \mathrm{HR}$ coating to be predicted, as presented in Fig. 2(b). For the HR calculation, we used a standard configuration of alternating quarter layers with refractive indices as listed in Table I.

The optical absorption expected in an $a-\mathrm{Si} / \mathrm{SiO}_{2} \mathrm{HR}$ coating based on the single-layer measurements after heat treatment at $500{ }^{\circ} \mathrm{C}$ is $354 \mathrm{ppm}$ at $1.55 \mu \mathrm{m}$ and $49 \mathrm{ppm}$ at $2 \mu \mathrm{m}$. The longer wavelength thus provides an improvement factor of $\approx 7.2$.

The optical absorption of an $a-\mathrm{Si} / \mathrm{SiO}_{2}$ HR multilayer coating as deposited was previously found to be $1042 \mathrm{ppm}$ at $1.55 \mu \mathrm{m}[36]$. Here, we measured the effect of heat treatment on the absorption of an HR sample from the same coating run. We carried out a series of measurements, equivalent to Fig. 2(a) at $1.55 \mu \mathrm{m}$. A maximum reduction of $57 \%$ was found for heat treatment at $450{ }^{\circ} \mathrm{C}$ resulting in a remaining absorption of $448 \mathrm{ppm}$. This is about $25 \%$ higher than the prediction from the single-layer measurements and thus in fairly good agreement. The improvement factor of 2.3, used in our further discussions, is based on the $448 \mathrm{ppm}$.

For precision measurement applications, the coating performance at low temperature is most relevant. Figure 3 shows the change in optical absorption with temperature normalized to the room temperature value. It was measured at 1.55 and $2 \mu \mathrm{m}$ for samples as deposited and heat treated at $400{ }^{\circ} \mathrm{C}$. The change in substrate response with temperature due to changing material parameters is $\leq 5.5 \%$ at both wavelengths and therefore negligible. At $2 \mu \mathrm{m}$, the absorption was corrected for the substrate absorption by using measurements of an uncoated sample as a function of temperature. The change in absorption for cooling the sample from room temperature to $47 \mathrm{~K}$ follows

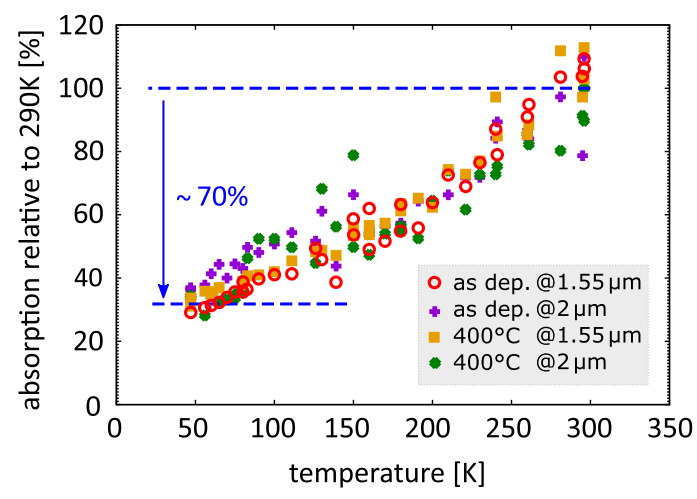

FIG. 3. Optical absorption measured on our $a$-Si single-layer coating at $1.55 \mu \mathrm{m}$ (as dep., red open circle; heat treated at $400{ }^{\circ} \mathrm{C}$, yellow square) and at $2 \mu \mathrm{m}$ (as dep., purple diamond; heat treated at $400{ }^{\circ} \mathrm{C}$, green solid circle), as a function of temperature. The measurements were corrected for substrate absorption and normalized to room temperature values.

the same trend for all four measurements showing a reduction of $\approx 70 \%$ (a factor 3.3 ). The absorption may further reduce at $20 \mathrm{~K}$.

The main error of our absorption measurements resulted from misalignment and was estimated to be $\leq 10 \%$. Another uncertainty may exist due to differences between properties of our substrate material and material parameters taken from the literature. This uncertainty is difficult to quantify and has not been considered in our error bars.

From all absorption results, we conclude a total reduction by a factor of about 55 for an $a-\mathrm{Si} / \mathrm{SiO}_{2} \mathrm{HR}$ coating resulting in $\approx 20 \mathrm{ppm}$ (for $k_{a-\mathrm{Si}}=3.5 \times 10^{-5}$ and negligible absorption in the $\mathrm{SiO}_{2}$ layers). The contributions are a factor of 2.3 from heat treatment, (at least) 3.3 from cooling and 7.2 when changing the wavelength from 1.55 to $2 \mu \mathrm{m}$. In the following section, we present mechanical loss measurements in order to discuss full exploitation of this significant optical absorption reduction.

Mechanical loss of a-Si.-Mechanical loss can be measured using the free amplitude decay of excited mechanical resonances $[29,48]$. To determine the loss of the $a$-Si coating, thin cantilevers were coated in the same deposition run as the absorption samples. A fused silica cantilever $(40 \mathrm{~mm} \times 5 \mathrm{~mm} \times 163 \mu \mathrm{m})$ was used for room temperature measurements, while a silicon cantilever $(35 \mathrm{~mm} \times 5 \mathrm{~mm} \times 67 \mu \mathrm{m})$ was used for cryogenic measurements. The different substrate materials were chosen to ensure sufficiently small contribution of the substrate loss to the measurement in the different temperature regimes.

The mechanical loss of the coating, $\phi_{a-\mathrm{Si}}$, can be calculated from a measurement of the mechanical loss of the coated cantilever $\phi_{\text {coated }}$ when the loss of the uncoated cantilever $\phi_{\text {uncoated }}$ is known:

$$
\phi_{a-\mathrm{Si}}=\frac{Y_{\text {cant }} \times t_{\text {cantilever }}}{\left(3 \times Y_{a-\mathrm{Si}} t_{a-\mathrm{Si}}\right)} \times\left(\phi_{\text {coated }}-\phi_{\text {uncoated }}\right),
$$



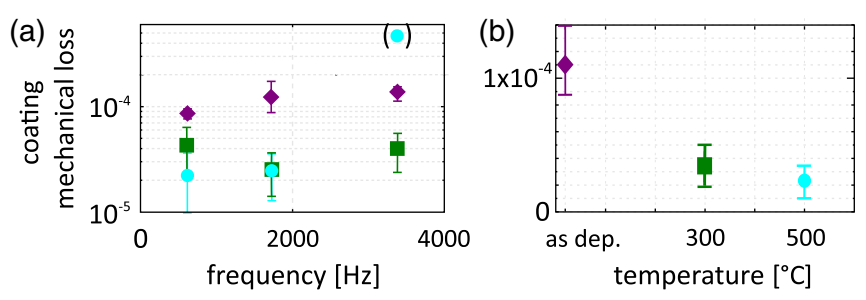

FIG. 4. (a) Coating mechanical loss at room temperature for the $a$-Si single layer after deposition (purple diamond) and after additional heat treatment at $300{ }^{\circ} \mathrm{C}$ (green square) and at $500{ }^{\circ} \mathrm{C}$ (blue circle). The mode marked with brackets was most likely influenced by interactions with the clamping structure [48,54] and was excluded from the analysis. (b) Same data as (a) but averaged over the results at different cantilever resonance frequencies.

where $Y$ is the Young's modulus $\left(\mathrm{SiO}_{2}: 73 \mathrm{GPa}\right.$ [49], $c$-Si: $160 \mathrm{GPa}$ [50], $a$-Si: $147 \mathrm{GPa}$ [51]) [52].

Figure 4(a) shows $\phi_{a-\mathrm{Si}}$ for three cantilever resonant frequencies for the coating as deposited and heat treated at 300 and at $500{ }^{\circ} \mathrm{C}$. The errors shown in Fig. 4 result from the spread in cantilever mechanical loss. It is possible that the heat treatment reduced the loss of the cantilever substrate. At the temperatures involved, this would have resulted in a small overestimation of the coating loss [53].

Figure 4(b) shows the same measurements but now averaged over the values obtained at different resonance frequencies. A significant improvement with heat treatment can be observed. The mechanical loss of our $a$-Si is approximately 4.5 times lower than that of previously measured $a$-Si [deposited via ion beam sputtering (IBS)] [29], both measured at room temperature after optimum heat treatment.

Figure 5 shows the mechanical loss as a function of temperature for our $a-\mathrm{Si}$ and, for comparison, for $a-\mathrm{Si}_{\mathrm{IBS}}$ taken from [29], at cantilever resonance frequencies of $1310 \mathrm{~Hz}$ (our $a$-Si) and $1444 \mathrm{~Hz}$ [previous $a$-Si (IBS)]. Neither coating was heat treated after deposition. The error bars of the mechanical loss measured on the $c$-Si cantilevers result from the standard deviation of several ring downs measured for each temperature step. At $20 \mathrm{~K}$, the mechanical loss of our $a$-Si is slightly below $0.2 \times 10^{-4}$ (Fig. 5). An almost identical value was found in [29] for their $a$-Si after optimum heat treatment. Thus, we suspect that the loss of our $a$-Si may also further reduce with heat treatment. This is subject of further investigations. For the time being, we used the value of $0.2 \times 10^{-4}$ for this work (see Table I).

Moving from $\mathrm{Ti}: \mathrm{Ta}_{2} \mathrm{O}_{5}$ to $a$-Si [Coating $C$ in Fig. 1] reduces thermal noise by $40 \%$ at $20 \mathrm{~K}$ compared to coating $B$ Fig. 1.

Optical absorption and mechanical loss of SiN.-Of the remaining thermal noise of coating $C,>99 \%$ is due to the $\mathrm{SiO}_{2}$ layers. This demonstrates the need to find an

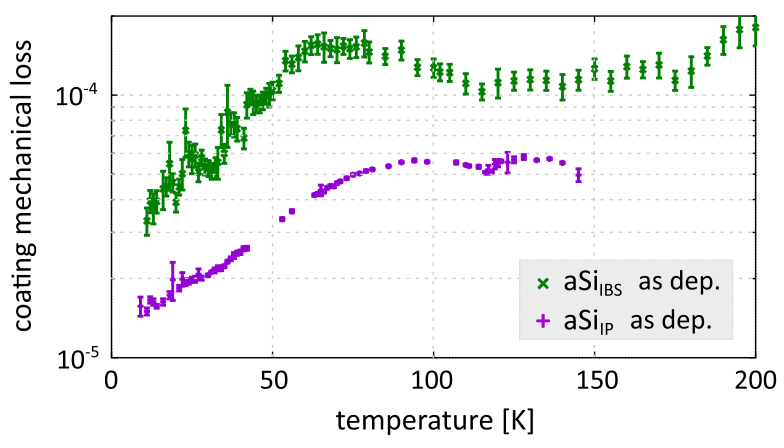

FIG. 5. Mechanical loss as a function of temperature for our $a$-Si single layer (lower, purple pluses) compared with previous measurements of $a$-Si (deposited via IBS, upper, green crosses) [29], both as deposited.

alternative low index material for use in combination with $a$-Si. A comparably low refractive index and low mechanical loss at low temperatures [32] (see Table I) make SiN an interesting candidate material. Previous work has shown an optical absorption coefficient of $\operatorname{SiN}$ of $\alpha=1.03 \mathrm{~cm}^{-1}$ $\left(k=1.2 \times 10^{-5}\right)$ at $1.55 \mu \mathrm{m}$ [38]. For the work presented in this Letter, we have measured the absorption of $\mathrm{SiN}$ at $2 \mu \mathrm{m}$ resulting in $k=4.3 \times 10^{-6}$. It should be noted that the optical absorption of $\mathrm{SiN}$ was measured at room temperature and may reduce with cooling.

The remarkably low mechanical loss of SiN (see Table I) combined with our results for $a$-Si allow for the coating $D$ in Fig. 1 to be realized. This coating has a a total optical absorption of $27 \mathrm{ppm}$ and thermal-noise factor of 12 lower than coating $B$ at $20 \mathrm{~K}$.

Conclusion.-For a long time, it was thought that the optical absorption of $a$-Si is too high for this material to be used in coatings for ultra-high precision metrology and sensing. In this Letter, we show a reduction in optical absorption of $a$-Si by a factor of 55 compared to previous results. This reduction was achieved by using a combination of heat treatment, final operation at low temperature, and a wavelength of $2 \mu \mathrm{m}$ instead of the more commonly used $1.55 \mu \mathrm{m}$. Combining $a$-Si with SiN to realize an HR multilayer coating results in an absorption of $27 \mathrm{ppm}$ at $2 \mu \mathrm{m}$ and $20 \mathrm{~K}$ with - compared to $\mathrm{SiO}_{2} / \mathrm{Ta}_{2} \mathrm{O}_{5}$ at $20 \mathrm{~K}$-a 12 times reduced thermal noise.

A factor of 12 in thermal-noise reduction is huge and solves the thermal-noise problem in gravitational-wave detectors. First of all, the thermal-noise reduction of our work would make it possible to achieve the design sensitivity of the low-frequency detector of the Einstein Telescope [8]. Furthermore, it might even lead to an increase of the average detection rate of $12^{3}$, if also other noise sources, in particular, quantum noise, can be reduced by similar large values.

Our result is a strong motivation for further research and development of amorphous $a-\mathrm{Si} / \mathrm{SiN}$ coatings. This 
includes investigation of the reasons for differences in optical and mechanical loss of $a$-Si deposited via IBS compared to RLVIP.

We are grateful for financial support from STFC (ST/ N005422/1), from the Royal Society (RG110331), from the ERC project MassQ (Grant Agreement No. 339897) and from the University of Glasgow. I. W. M. is supported by a Royal Society Research Fellowship. We are grateful to the International Max Planck Partnership for Measurement and Observation at the Quantum Limit for support, and we thank our colleagues in the LSC and Virgo Collaborations and within SUPA for their interest in this work. In particular we would like to thank Dr. A. Markosyan and Professor S. Chao for their helpful input and Dr. S. Agon for valuable support with annealing the samples.

This paper is LIGO Document No. LIGO-P1800004.

*iain.martin@glasgow.ac.uk

[1] B. P. Abbott et al., Phys. Rev. Lett. 116, 061102 (2016).

[2] B. P. Abbott et al., Phys. Rev. Lett. 116, 241103 (2016).

[3] B. P. Abbott et al., Phys. Rev. Lett. 118, 221101 (2017).

[4] B. P. Abbott et al., Phys. Rev. Lett. 119, 141101 (2017).

[5] B. P. Abbott et al., Phys. Rev. Lett. 119, 161101 (2017).

[6] Y. Levin, Phys. Rev. D 57, 659 (1998).

[7] G. M. Harry, A. M. Gretarsson, P. R. Saulson, S. E. Kittelberger, S. D. Penn, W. J. Startin, S. Rowan, M. M. Fejer, D. R. M. Crooks, G. Cagnoli, J. Hough, and N. Nakagawa, Classical Quantum Gravity 19, 897 (2002).

[8] S. Hild, S. Chelkowski, A. Freise, J. Franc, N. Morgado, R. Flaminio, and R. DeSalvo, Classical Quantum Gravity 27, 015003 (2010).

[9] M. Abernathy et al., Report No. ET-0106C-10 (2011), https://tds.ego-gw.it/ql/?c=7954.

[10] R. Adhikari et al. (LIGO III Blue ConceptLIGO), Technical Document LIGO-G1200573 (2012), dcc.ligo.org/LIGOG1200573-v1/public.

[11] I. Ushijima, M. Takamoto, M. Das, T. Ohkubo, and H. Katori, Nat. Photonics 9, 185 (2015).

[12] N. Huntemann, C. Sanner, B. Lipphardt, C. Tamm, and E. Peik, Phys. Rev. Lett. 116, 063001 (2016).

[13] D. G. Matei et al.,Phys. Rev. Lett. 118 (2017).

[14] M. Granata et al., Opt. Lett. 38, 5268 (2013).

[15] B. P. Abbott et al., Phys. Rev. Lett. 116, 131103 (2016).

[16] R. Flaminio, J. Franc, C. Michel, N. Morgado, L. Pinard, and B. Sassolas, Classical Quantum Gravity 27, 084030 (2010).

[17] I. W. Martin et al., Classical Quantum Gravity 31, 035019 (2014).

[18] I. W. Martin et al., Classical Quantum Gravity 26, 155012 (2009).

[19] G. M. Harry et al., Classical Quantum Gravity 24, 405 (2007).

[20] R. Nawrodt et al., J. Phys. Conf. Ser. 122, 012008 (2008).

[21] D. F. McGuigan, C. C. Lam, R. Q. Gram, A. W. Hoffman, D. H. Douglass, and H. W. Gutche, J. Low Temp. Phys. 30, 621 (1978).
[22] W. Winkler, K. Danzmann, A. Rüdiger, and R. Schilling, Phys. Rev. A 44, 7022 (1991).

[23] S. Rowan, R. L. Byer, M. M. Fejer, R. Route, G. Cagnoli, D. R. M. Crooks, J. Hough, P. H. Sneddon, and W. Winkler, Proc. SPIE Int. Soc. Opt. Eng. 4856, 292 (2003).

[24] R. Schnabel et al., J. Phys. Conf. Ser. 228, 012029 (2010).

[25] G. D. Cole, W. Zhang, M. J. Martin, J. Ye, and M. Aspelmeyer, Nat. Photonics 7, 644 (2013).

[26] G. D. Cole et al. Optica 3, 647 (2016).

[27] J. Steinlechner, I. W. Martin, A. Bell, G. Cole, J. Hough, S. Penn, S. Rowan, and S. Steinlechner, Classical Quantum Gravity 32, 105008 (2015).

[28] X. Liu, D. R. Queen, T. H. Metcalf, J.E. Karel, and F. Hellman, Phys. Rev. Lett. 113, 025503 (2014).

[29] P. G. Murray, I. W. Martin, K. Craig, J. Hough, R. Robie, S. Rowan, M. R. Abernathy, T. Pershing, and S. Penn, Phys. Rev. D 92, 062001 (2015).

[30] L. Pinard et al., Appl. Opt. 56, C11 (2017).

[31] J. Steinlechner, I. W. Martin, R. Bassiri, A. Bell, M. M. Fejer, J. Hough, A. Markosyan, R. K. Route, S. Rowan, and Z. Tornasi, Phys. Rev. D 93, 062005 (2016).

[32] X. Liu, T. H. Metcalf, Q. Wang, and D. M. Photiadis, in Proceedings of Symposium A: Amorphous and Polycrystalline Thin-Film Silicon Science and Technology (MRS, Pittsburgh, PA, 2007), Vol. 989, p. 511.

[33] D. R. Southworth, R. A. Barton, S. S. Verbridge, B. Ilic, A. D. Fefferman, H. G. Craighead, and J. M. Parpia, Phys. Rev. Lett. 102, 225503 (2009).

[34] I. W. Martin et al., Classical Quantum Gravity 27, 225020 (2010).

[35] Norcada, Inc., Canada, www.norcada.com.

[36] J. Steinlechner, A. Khalaidovski, and R. Schnabel, Classical Quantum Gravity 31, 105005 (2014).

[37] S. Chao, H. Pan, C. Lee, and L. Kuo, LIGO Technical Document, dcc.ligo.org/LIGO-G1300171/public.

[38] J. Steinlechner, C. Krüger, I. W. Martin, A. Bell, J. Hough, H. Kaufer, S. Rowan, R. Schnabel, and S. Steinlechner, Phys. Rev. D 96, 022007 (2017).

[39] J. Steinlechner, C. Krüger, I. W. Martin, A. Bell, J. Hough, H. Kaufer, S. Rowan, R. Schnabel, and S. Steinlechner, Phys. Rev. D 96, 022007 (2017).

[40] J. Steinlechner, I. W. Martin, J. Hough, C. Krueger, S. Rowan, and R. Schnabel, Phys. Rev. D 91, 042001 (2015).

[41] Corning, Inc., USA, http://www.corning.com.

[42] D. B. Leviton and B. J. Frey, Proc. SPIE Int. Soc. Opt. Eng. 6273, 62732K (2006).

[43] Tafelmaier Dünnschicht-Technik GmbH, Rosenheim, Germany, http://www.tafelmaier.de.

[44] S. Schlichtherle, G. N. Strauss, H. Tafelmaier, D. Huber, and H. K. Pulker, Vakuum in Forschung und Praxis 17, 210 (2005).

[45] A. L. Alexandrovski, M. M. Fejer, A. Markosyan, and R. Route, Proc. SPIE Int. Soc. Opt. Eng. 71930, 71930D (2009).

[46] It has been shown for $a$-Si deposited via IBS that heat treatment in air and in vacuum results in identical optical absorption, and heat treatment for more than one hour does not further reduce the optical absorption [31]. 
[47] https://github.com/sestei/dielectric.

[48] S. Reid, G. Cagnioli, D. R. M. Crooks, J. Hough, P. Murray, S. Rowan, M. M. Fejer, R. Route, and S. Zappe, Phys. Rev. A 351, 205 (2006).

[49] R. Brückner, J. Non-Cryst. Solids 5, 123 (1970).

[50] Y. S. Touloukian and E. H. Buyco, Thermo-physical Properties of Matter (Plenum, New York, 1970).

[51] M. R. Abernathy, Ph.D. thesis, University of Glasgow (2012).
[52] Room temperature values have been used for $Y$. For $c$-Si, the effect of cooling is negligible [48]. Here, we assume the same for $a-\mathrm{Si}$.

[53] B.S. Lunin, Physical and Chemical Bases for the Development of Hemispherical Resonators for SolidState Gyroscopes, (Moscow Aviation Institute, Moscow, 2005).

[54] I. W. Martin, Ph.D. thesis, University of Glasgow (2009). 